\title{
The Aboriginal Maternal and Infant Health Service: a decade of achievement in the health of women and babies in NSW
}

\author{
Elisabeth Murphy ${ }^{\mathrm{A}, \mathrm{B}}$ and Elizabeth Best ${ }^{\mathrm{A}}$ \\ A Maternity, Children and Young People's Health Branch, \\ NSW Ministry of Health \\ ${ }^{\mathrm{B}}$ Corresponding author.Email: lmurp@doh.health.nsw.gov.au
}

\begin{abstract}
The Aboriginal Maternal and Infant Health Service was established to improve the health of Aboriginal women during pregnancy and decrease perinatal morbidity and mortality for Aboriginal babies. The Service is delivered through a continuity-of-care model, where midwives and Aboriginal Health Workers collaborate to provide a high quality maternity service that is culturally sensitive, women centred, based on primary health-care principles and provided in partnership with Aboriginal people. An evaluation of the Service found that the program is achieving its goals in relation to the provision of antenatal and postnatal care and has demonstrated improvements in perinatal morbidity and mortality rates.
\end{abstract}

In 2009, 96439 babies were born in New South Wales (NSW); of these, 2931 (3\%) were born to mothers who identified as Aboriginal. ${ }^{1}$ Higher rates of perinatal morbidity and mortality are found in Aboriginal woman compared with non-Aboriginal women. These higher rates are associated with poor health status, underuse of antenatal and postnatal services, a high adolescent birth rate and the social, economic and political factors affecting many women in Aboriginal communities. ${ }^{2,3}$

In 1998, the Aboriginal Perinatal Mortality Project was established to address the high rate of Aboriginal perinatal mortality in NSW. The Project was a response to the NSW Maternal and Perinatal Committee's review of Aboriginal perinatal deaths in $1998 .{ }^{2}$ The review found that $59 \%$ of Aboriginal perinatal deaths were stillbirths and $41 \%$ were neonatal deaths (Table 1). In 2000, the NSW Aboriginal perinatal mortality rate was 17.9 per 1000 births compared to the non-Aboriginal rate of 9.7 per 1000 births. The percentage of low birthweight Aboriginal babies (a risk factor for perinatal morbidity and mortality) in the same year was $11.9 \%$, which was almost twice the nonAboriginal rate of $6.4 \% .^{2}$

The review revealed that a large number of the neonatal deaths were related to extreme prematurity. The review found that, of the 32 perinatal deaths in Aboriginal babies in 1998, 34\% (11 deaths) of the mothers had no, or two or fewer, antenatal care visits. As antenatal care was not recorded in an additional seven records, the true proportion of women who did not have antenatal care could have been as high as $56 \% .^{2}$ The under-utilisation of antenatal and postnatal services by Aboriginal women results in inadequate management of complications during pregnancy and the perinatal period and leads to increased perinatal mortality and morbidity. ${ }^{2}$ Figure 1 shows a reduction of perinatal deaths from 1990 to 2006 and increased access to antenatal care before 20 weeks from 1994 to 2006 .

In 1998, the Aboriginal Perinatal Mortality Project found that, to improve the health of Aboriginal mothers and babies, creative public health strategies were required: to increase the self-esteem of Aboriginal women; to have strong and cohesive Aboriginal communities with raised standards of living; and to increase access by Aboriginal women to antenatal services by making maternity services more accessible and culturally appropriate.

As a result, a model based on the findings of the Aboriginal Perinatal Mortality Project was developed. This model was called the NSW Aboriginal Maternal and Infant Health

Table 1. Perinatal deaths among Aboriginal babies in NSW, 1998

\begin{tabular}{lccc}
\hline $\begin{array}{l}\text { Gestational age } \\
\text { Weeks }\end{array}$ & $\begin{array}{c}\text { Stillbirths } \\
\mathbf{n}\end{array}$ & $\begin{array}{c}\text { Neonatal deaths } \\
\mathbf{n}\end{array}$ & $\begin{array}{c}\text { Total } \\
\mathbf{N}\end{array}$ \\
\hline & 6 & 8 & 14 \\
$20-27$ & 3 & 1 & 4 \\
$32-31$ & 5 & 1 & 6 \\
$37-41$ & 5 & 3 & 8 \\
Total & 19 & 13 & 32 \\
\hline
\end{tabular}

Source: The NSW Aboriginal perinatal health report. Sydney: NSW Department of Health; 2003. 

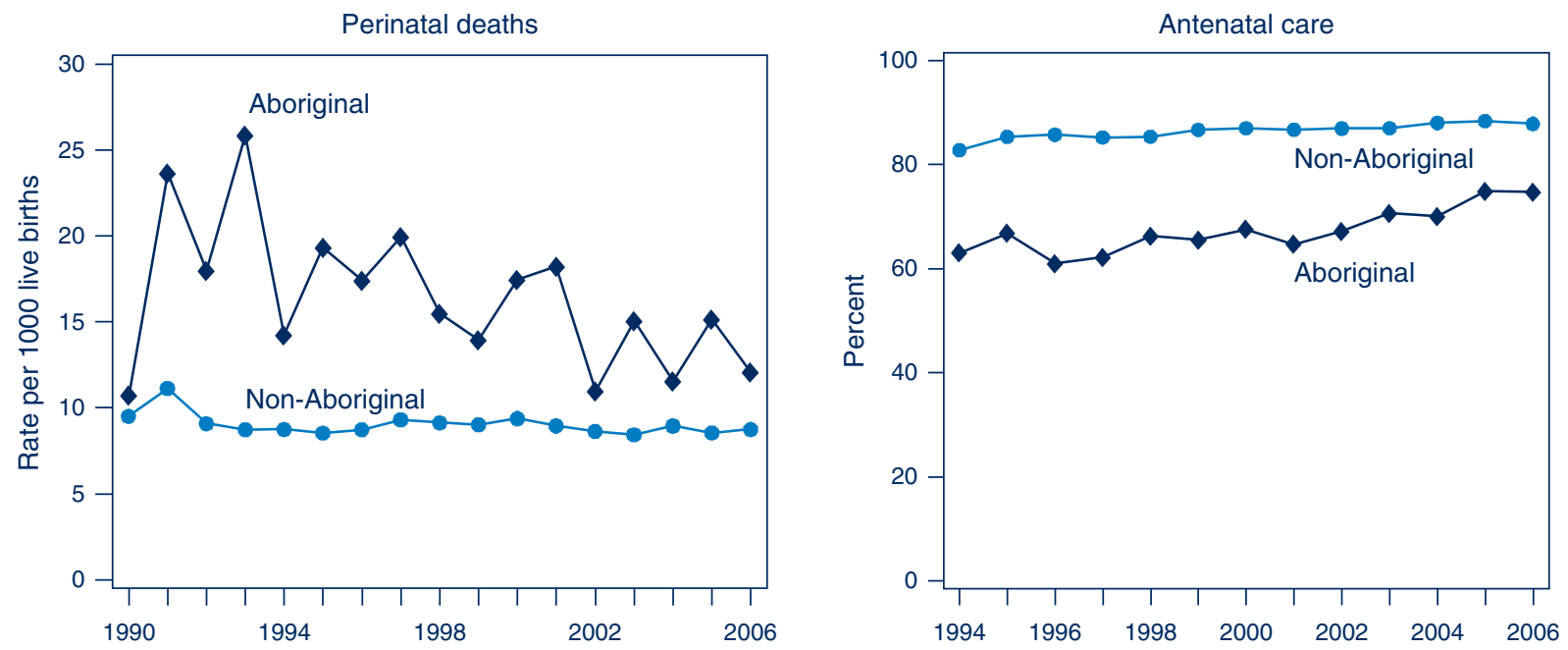

Figure 1. Comparison for Aboriginal and non-Aboriginal mothers in NSW of the rate of perinatal deaths of babies for the period 1990 to 2006 and of the timing of the first antenatal visit before 20 weeks gestation for the period 1994 to 2006.

Source: NSW Midwives Data Collection (HOIST). Centre for Epidemiology and Research, NSW Ministry of Health.

Strategy (now known as the Aboriginal Maternal and Infant Health Service [AMIHS]). ${ }^{4}$ This paper describes a decade of the development, evaluation and expansion of a service designed to improve maternity and infant health outcomes for Aboriginal women and babies. This service is the first of its kind in NSW.

\section{Model of care}

AMIHS was funded by NSW Health in December 2000 and implemented in 2001. The goal was to improve the health of Aboriginal women during pregnancy and decrease perinatal morbidity and mortality for Aboriginal babies and their mothers in NSW. A continuity-of-care model was developed in which midwives and Aboriginal Health Workers work together to provide a high-quality maternity service. AMIHS is a culturally appropriate maternity service; its philosophy is based on cultural respect, social justice, participation, equality, access, learning and collaboration. The guiding principles that make the Service unique include taking a broad social view of health, forming effective partnerships with Aboriginal communities, working within a primary healthcare framework and providing women-centred care.

AMIHS midwives and Aboriginal Health Workers provide antenatal and postnatal care, from as early as possible after conception up to 8 weeks postpartum. The care is provided in the community but is linked into mainstream maternity services to ensure that risk management and education are available to AMIHS teams.

\section{Community consultation}

Community consultation is critical in AMIHS. All associated programs are designed in consultation and in line with the local context and needs, community relationships and community and health sector expectations. This ensures that the service is located in an appropriate setting and is accessible to the Aboriginal community. Flexible service delivery and the provision of transport are essential for access to the AMIHS and to the services to which patients are referred. AMIHS services can vary depending on the needs of the local community and the skills and expertise of the staff.

AMIHS takes a holistic approach to Aboriginal women's health. Local programs encourage innovative community development projects to engage and empower Aboriginal women and their communities. Health promotion is achieved through Aboriginal Health Workers using community development strategies in partnership with other agencies.

\section{Partnering with other services}

Effective local Aboriginal health partnerships and collaboration with other services are important to ensure the best outcomes for families. It is particularly important for AMIHS to have strong links with the local Aboriginal Community Controlled Health Service and other nongovernment and government agencies. This collaborative approach aims to ensure the sustainability of the program. Effective collaboration also includes consultation and referral with health and other services, such as general practice, to assure high-quality, integrated pregnancy care.

An example of effective collaboration is the link established between AMIHS and the Brighter Futures program. The Brighter Futures program is an early intervention program of the NSW Department of Family and Community Services, Community Services Division, that provides support early in a child's life that is tailored to meet the needs of each family. Its aim is to keep children out of the child protection system and to improve child wellbeing by supporting families. This link means that eligible AMIHS 
families can more easily access services such as child care, parenting programs and home visiting.

\section{Transition of care}

The smooth transition of care to child and family health nursing services ensures continuity of care for the child up until school age. Child health and developmental checks at specific milestones in early life aim to ensure that the child reaches school age ready to learn. NSW Health is implementing the Building Strong Foundations for Aboriginal Children, Families and Communities program in a number of areas. This program is based on the AMIHS model with a child and family health nurse and an Aboriginal health education officer providing primary early childhood health care to families up until the child goes to school. Where Building Strong Foundations programs are located near an AMIHS, the transition of care for a family is seamless from the maternity service to the child and family health service.

\section{Training and Support Unit}

A statewide Training and Support Unit is another element of the AMIHS model. Its aim is to provide professional development and support, reduce the isolation of AMIHS staff and develop networks. It successfully promotes collaboration and partnerships between AMIHS programs, including providing locally based training for staff in each AMIHS network. The unit also organises statewide conferences in order to share knowledge, resources and to provide networking opportunities among health workers in AMIHS across the state who can often be professionally and geographically isolated.

AMIHS adheres to NSW Health policies and procedures and legislation, including those that protect and promote the safety, welfare and wellbeing of children. Programs provide data for statewide Key Performance Indicators and submit quarterly and annual reports at the end of each financial year.

\section{Evaluation of the Aboriginal Maternal and Infant Health Service}

As part of the implementation of AMIHS in 2000, external consultants were engaged to plan the evaluation of the Service. The evaluation was longitudinal and data were collected at four time periods over 3 years. The aim was to measure the extent to which AMIHS was achieving its goals and to identify its strengths. Based on these findings, the evaluation report then made recommendations for future improvements. ${ }^{4}$

\section{Results of the evaluation}

The evaluation showed that AMIHS was improving maternity services and outcomes for Aboriginal women and that Aboriginal women trusted and supported the service provided. ${ }^{4}$

The evaluation showed that AMIHS had achieved the following outcomes for Aboriginal mothers and babies:

- increased proportion of women who attended their first antenatal visit before 20 weeks gestation $(65 \%$ before the Service began versus $78 \%$ in 2004)(OR 1.2; 95\% CI $1.01-1.4 ; p=0.03)$.

- decreased rate of low birthweight babies (13\% versus $12 \%$ after the Service). The difference is not statistically significant.

- decreased proportion of preterm births $(20 \%$ versus 11\%; OR 0.5; 95\% CI 0.4-0.8-1.4; $p<0.001)$.

- decrease in perinatal mortality from 1996-2000 (20.4 per 1000 births) compared to 2001-2003 (14.4 per 1000 births) in Local Government Areas where AMIHS was located. The difference is not statistically significant owing to small numbers.

- improved breastfeeding rates from 67\% initiating breastfeeding and 59\% still breastfeeding at 6 weeks in 2003 , to $70 \%$ initiating breastfeeding and $62 \%$ still breastfeeding at 6 weeks in 2004 .

The evaluation also showed that Aboriginal women were very satisfied with AMIHS programs. Home visiting, inclusion of an Aboriginal worker on the team and reminders about and transport to antenatal appointments were the most important aspects cited.

The evaluation highlighted the importance of the Training and Support Unit in providing support and development opportunities for Service staff. The Unit linked Aboriginal Health Workers to a maternity and infant health training course and a number of graduates enrolled in a Bachelor of Midwifery program to become qualified midwives.

The evaluation made recommendations for improvement, including:

- that access to child and family health services targeting Aboriginal families be improved.

- that smoking cessation programs be a priority.

- that the inclusion of an Aboriginal worker in the Training and Support Unit be considered.

AMIHS continues to make an impact on Aboriginal women accessing antenatal care (Figure 2).

\section{Expansion of the Aboriginal Maternal and Infant Health Service}

In 2007, additional funding was provided to expand AMIHS from 7 programs in 6 former NSW Area Health Services to over 30 programs with many more sites across NSW (Figure 3). The former Department of Community Services (now Department of Family and Community Services) contributed joint funding with NSW Health for 2 years to support the statewide expansion and to establish a priority referral pathway between the AMIHS and the 


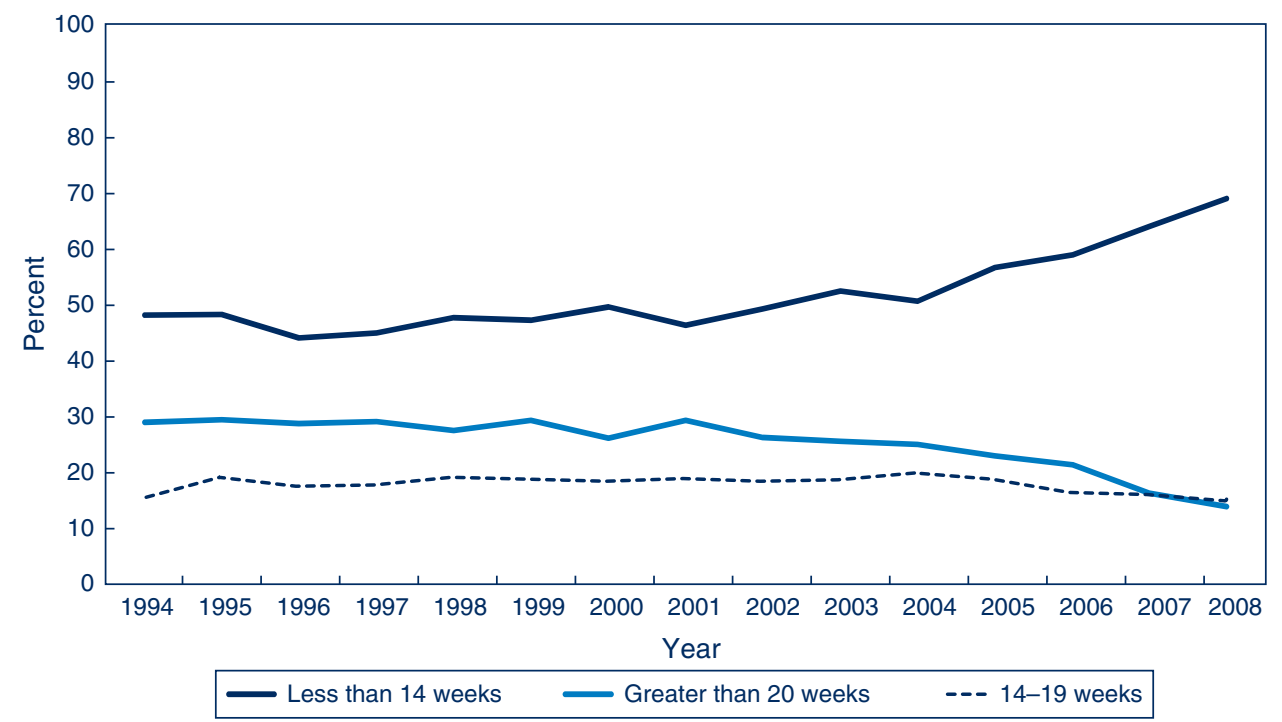

Figure 2. Trends in gestational age of Aboriginal babies in NSW at the start of antenatal care for the period 1994-2008.

Source: NSW Midwives Data Collection (HOIST). Centre for Epidemiology and Research, NSW Department of Health.

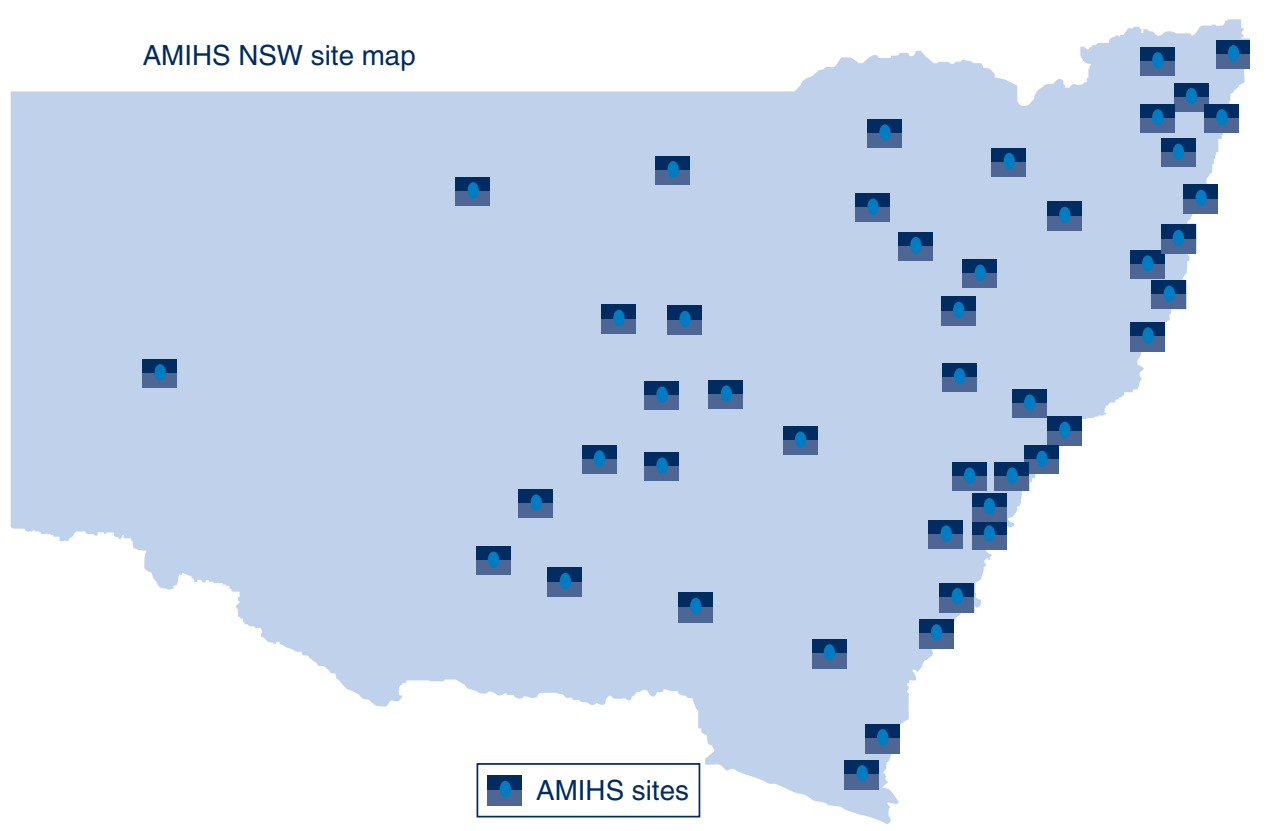

Figure 3. Locations of Aboriginal Maternal and Infant Health Service (AMIHS) programs in NSW, 2012.

Brighter Futures early intervention program. AMIHS now covers approximately $75 \%$ of Aboriginal births.

With the expansion of AMIHS and the implementation of Building Strong Foundations, the Training and Support Unit has been restructured into the Training and Support Unit for Aboriginal Mothers, Babies and Children. This Unit consists of a number of educators and other staff, including Aboriginal health educators. The Training and Support Unit is situated in the Rural Directorate of the Health Education and Training Institute.
As part of the expansion of services, funding was also provided for AMIHS resources. These included a service delivery model and workforce and recruitment strategy, the Strong Women Strong Babies Pregnancy Diary and safe sleeping brochures for consumers. Within the Closing the Gap initiatives, a number of new programs are being implemented to support AMIHS programs. These programs include secondary mental health, and drug and alcohol services in selected AMIHS programs and the Quit for new life smoking cessation in pregnancy program in all AMIHS sites. 
The Aboriginal Maternity Data Collection, a de-identified database from the Perinatal Data Collection, was set up to support the data management and evaluation of AMIHS. Work is continuing on the collection to ensure that the data systems for AMIHS are robust to assist in the current evaluation of AMIHS.

\section{Conclusions}

The evaluation of AMIHS demonstrated that the program is successfully meeting its goal to improve the health outcomes of Aboriginal mothers and babies by providing culturally appropriate maternity care.

The evaluation highlighted the strengths in the program, including the partnerships between the Aboriginal Health Workers and midwives working together in a primary health-care model to provide continuity of care. It is delivered in the community by a highly committed skilled team who have strong relationships with both the community and the health services. The retention of staff and the trust between the AMIHS team and the women in their care are impressive.

Recommendations to strengthen the current model have subsequently been addressed through the implementation of a culturally appropriate child health program (Building Strong Foundations), the implementation of the Aboriginal specific Quit for new life smoking cessation program and the restructuring of the Training and Support Unit to include Aboriginal health educators.

The next evaluation of AMIHS will again determine whether the program is achieving its goals and identify its strengths 10 years after its commencement.

\section{References}

1. Centre for Epidemiology and Research. New South Wales Mothers and Babies 2009. Sydney: NSW Ministry of Health, 2011. Available at: http://www.health.nsw.gov.au/pubs/2011/ pdf/mothers_babies_2009.pdf (Cited 8 October 2011).

2. Health NSW. The NSW Aboriginal perinatal health report. Sydney: NSW Department of Health; 2003. Available at: http://www.health.nsw.gov.au/pubs/2003/pdf/abl_peri.pdf (Cited 8 October 2011).

3. Laws P, Li Z, Sullivan EA. Australia's mothers and babies 2008. Perinatal statistics series no. 24. Cat. no. PER 50. Canberra: AIHW.

4. Health NSW. Aboriginal Maternal and Infant Health Strategy. Evaluation. Final report 2005. Available at: http://www.health. nsw.gov.au/pubs/2006/pdf/evaluation_maternal.pdf (Cited 8 October 2011). 